\title{
Influence of early post-burn enteral nutrition on clinical outcomes of patients with extensive burns
}

\author{
Guozhong Lu, ${ }^{1}$ Jiren Huang, ${ }^{1, *}$ Junjie Yu, ${ }^{1}$ Yugang Zhu, ${ }^{1}$ Liangliang Cai, ${ }^{1}$ Zaiqiu Gu${ }^{1}$ and Qinghe Su${ }^{1}$ \\ 'Department of Burn and Plastic Surgery, The Third People's Hospital Affiliated, School of Medicine, Nantong University, Wuxi, Jiangsu 214041, China
}

(Received 26 July, 2010; Accepted 4 September, 2010; Published online 18 February, 2011)

\begin{abstract}
Sepsis commonly occurs in severe post-burn patients, often resulting in death. We aimed to evaluate the influence of early enteral feeding on outcomes in patients with extensive burns, including infection incidence, healing and mortality. We retrospectively reviewed 60 patients with extensive burns, 35 who had received early enteral nutrition and $\mathbf{2 5}$ who had received parenteral nutrition. Average healing time, infection incidence and mortality were clinically observed. Hemoglobin and serum albumin were monitored weekly in both groups during treatment. Causative organisms were identified in patients with sepsis. Infection incidence was significantly less in the enteral nutrition group than the parenteral nutrition group $(17.1 \%$ vs $44.0 \% ; p=0.023)$; and latency duration was longer in the enteral nutrition group than in the parenteral nutrition group $(30.5 \pm 4.7$ days vs $14.5 \pm 2.3$ days; $p<0.001)$. Duration of antibiotic therapy of the enteral nutrition group was significantly shorter than that of the parenteral nutrition group $(12.5 \pm 3.0$ days vs $19.8 \pm 3.6$ days; $p<0.001)$. Mean hemoglobin results $(10.1 \pm 1.3 \mathrm{~g} / \mathrm{L}$ vs $8.3 \pm 1.5 \mathrm{~g} / \mathrm{L} ; p<0.001)$ and serum albumin results $(44.7 \pm 5.7 \mathrm{~g} / \mathrm{L}$ vs $36.2 \pm 6.9 \mathrm{~g} / \mathrm{L} ; p<0.001)$ of enteral nutrition and parenteral nutrition groups, respectively, provided an overview of systemic nutrition and protein metabolism, suggesting higher systemic nutrition and protein synthesis in enteral nutrition group than in parenteral nutrition group. Risk of post-burn infection is reduced in burn patients who are supported by earliest possible enteral nutrition.
\end{abstract}

Key Words: enteral nutrition, enterogenic infection, extensive burns, hypermetabolism

S epsis is a common complication of severe post-burn and is $\checkmark$ a leading cause of death in burn patients. ${ }^{(1)}$ Although the primary infection can be found in most burn cases, enterogenic infection due to the presence of permanent intestinal bacteria such as Gram-negative bacilli, enterococcus and Candida albicans may be observed in some burn patients. Bacterial translocation from the intestine has been shown to be the primary pathogenesis of most enterogenic infections and, in burns, may contribute to the development of sepsis. Bacteria may translocate across the mucosal barrier under the following conditions: mechanical destruction of the intestinal mucosa, reduced or abnormal immune function, and overgrowth of enteric bacteria. ${ }^{(2)}$ If these conditions are present in extensive burns accompanied by shock, it may result in intestinal ischemia, increased permeability of the intestinal mucosa, intestinal barrier failure, and burn-induced distant organ dysfunction. (2) The combination of reduced post-burn immune function, destruction of intestinal microecology, and dysbacteriosis caused by dysregulation of nutritional metabolism, complicated by the use of broad-spectrum antibiotics and increased colonization of intestinal bacteria, can lead to enterogenic infection and, in some cases, progress to septicemia. ${ }^{(2-5)}$

Over the last twenty years, clinicians have believed that providing an adequate supply of nutrients can reduce the increased metabolic demands associated with burns and help reduce septic morbidity. In the last ten years, a variety of studies have indicated that feeding through the gastrointestinal (GI) tract immediately after extensive burns can depress hypermetabolism, effectively prevent atrophy or injury of the GI mucosa, alleviate damage to the mucosal barrier function of the GI tract, and reduce bacterial translocation from the intestine, thereby decreasing enterogenic infection and sepsis. ${ }^{(6-10)}$

We, therefore, hypothesized that feeding through the GI tract immediately after patients sustained extensive burns could depress hypermetabolism, prevent intestinal mucosa atrophy, alleviate damage to the intestinal barrier function, reduce bacterial translocation from the intestine, and ultimately reduce the risk of enterogenic infection. In this study, we aimed to evaluate the effect of early enteral feeding, as compared to parenteral feeding, on several outcomes, including the translocation of intestinal organisms, GI mucosal injury and the incidence of enterogenic infection in patients with extensive burns.

\section{Materials and Methods}

We conducted a retrospective review of patients with extensive burns who had received treatment between 2002 and 2007 at the burn unit of The People's Hospital No. 3, Wuxi, Jiangsu, China. This study was approved by the Ethics Committee of the Third People's Hospital affiliated School of Medicine, Nantong University.

Patients who had burns of $60 \%$ or more of total body surface area (TBSA) and $30 \%$ or more full-thickness burn area were included in this study. Burn patients who had significant abdominal distension, diarrhea, stress ulcer bleeding, or combined digestive tract injuries were excluded from this study. A total of 60 burn patients were included in this study. While hospitalized due to extensive burns, patients had been treated with either early enteral nutrition or parenteral nutrition only as determined by clinicians. When the records of the 60 patients who met the inclusion criteria were retrospectively reviewed, patients were assigned to an early enteral nutrition group (EN group) or a parenteral nutrition group (PN group) according to the post-burn treatment received. The EN group included 35 patients who had received early post-burn enteral nutrition and the PN group included 25 patients who had received parenteral nutrition only and had not received early enteral nutrition as part of post-burn care.

The EN group received enteral feeding as early as possible after

*To whom correspondence should be addressed.

E-mail: huangjiren2009@yeah.net 
admission to the burn unit, usually within $1 \mathrm{~h}$ after admission unless the patient had food intake before the burn, in which case enteral feeding was initiated 4 to $6 \mathrm{~h}$ later. An indwelling nasogastric tube was inserted at the same time as patients received debridement and anti-shock treatment. In the early stage of postburn, enteral nutrition was delivered nasally as small amounts of warm fluid; the fluid amount was limited to about $100 \mathrm{ml}$ once every 3 to $4 \mathrm{~h}$ while offering an oral antiemetic (e.g., domperidone tablets) to improve gastric emptying. Water intake was calculated and adjusted based on the size of the wounds, whether or not patients underwent tracheotomy, whether air-fluidized beds were used, wound evaporation conditions, and patients' fluid discharge. Food intake was increased and its quality altered depending on presence of any symptoms of intestinal intolerance such as abdominal distension or gastralgia, the recovery of intestinal peristalsis or until patients' nutritional requirements could be completely fulfilled and nitrogen balanced.

For the enteral feeding group, the basic principles of nutritional supplementation for post-burn patients were followed. In the early stage of treatment, the patients received both enteral and parenteral nutritionsince tgastrointestinal function is dramatically reduced immediately after burns, and enteral feeding alone might not meet patients' caloric and nutritional needs. In the middle or later stages of treatment, patients were given only nasal or oral enteral nutrition. Enteral feeding was generally administered every 3 to $4 \mathrm{~h}$ for 4 to 8 weeks.

The formula for early enteral nutrition consisted of rice water (e.g., nutrient-rich liquid reserved from cooking rice or millet in water) and prepared enteral formulas, including Peptisorb (Nutricia Clinica, Auckland, New Zealand) and Nutrison Powder (Milupa Gmbh, Fulda, Germany). In the middle treatment stage, the major components of enteral nutrition were Nutrison Powder and Nutrison Fibre (Milupa Gmbh, Fulda, Germany). The diet was transitioned to a general consolidated diet in the advanced stage of treatment until recovery. The caloric needs of adult burn patients were estimated based on the following formula: $[\mathrm{kJ} / \mathrm{d}=4184 \times$ body surface area $\left(\mathrm{m}^{2}\right)+104.6 \times \%$ TBSA]; body surface area $\left(\mathrm{m}^{2}\right)=$ [height $(\mathrm{m})-0.6] \times 1.5$. We followed current clinical practice, keeping the total number of calories slightly lower than the calculated value to achieve better results in glucose control, prevention of infection-related complications, and reduction of overall hospital stays. ${ }^{(11)}$

The patients in the PN group abstained from food for three days during the shock period after injury, and then received parenteral nutrition only for 2 to 3 weeks. Three liters of fluid were infused at a constant rate for $24 \mathrm{~h}$, including glucose, amino acids, fat emulsion, electrolytes, water-soluble vitamins, fat-soluble vitamins, and trace elements. Parenteral nutrition was intended to help maintain tension within the GI tract, reduce injury and accelerate functional recovery of the GI tract. Juices were fed gradually after the patient recovered from shock and intestinal peristalsis resumed. Food intake was adjusted according to patients' individual condition. Generally speaking, normal nutrition through the GI tract was not resumed until one month after injury, and parenteral nutrition was still provided as a supplement.

All burn patients in this study received a single broad-spectrum antibiotic during the shock period immediately post-burn (48-72 h), including third-generation cephalosporin, sulperazon, cefepime, and ceftazidime. Antibiotic therapy was upgraded 2 days after the shock period or 3 days post-burn to an empirical combination of antibiotic therapies. Termination of antibiotic therapy was empirically determined by the physician according to the condition of each individual patient. Drug therapy was adjusted according to results of wound cultures during antibiotic therapy. Drugs were withdrawn when wound cultures showed no pathogenic bacteria.

The average healing time, the incidence of infection, the timing of infection, morbidity and mortality in both groups were recorded. The time to first post-burn infection (latency) and primary infec- tion focus were observed in cases with systemic infection. Tissue biopsy samples were cultured in the early post-burn period to identify infective organisms and again later to monitor the effectiveness of antibiotic therapy; quantitative cultures were done to indicate degree of colonization.

In both groups, an overview of systemic nutrition and protein metabolism was obtained by monitoring patients' hemoglobin and serum albumin values weekly for 4 weeks during the course of treatment. Average healing time, morbidity and mortality were clinically observed and differences between EN and PN groups were calculated and recorded.

Our transfusion policy included transfusing those patients with hemoglobin less than $12 \mathrm{~g} / \mathrm{L}$ after skin grafting. Transfusions of whole blood and plasma were administered as needed, nonroutinely (data not included).

Statistical Analysis. All statistical analyses were performed using SPSS 15.0 statistics software (SPSS Inc., Chicago, IL). Group comparisons for normally distributed continuous variables were made by independent two-sample $t$ tests. Chi-square test or Fisher's exact test was used to compare differences for categorical variables. Mann-Whitney $U$ tests were performed to determine group differences in total liquid volume. Normally distributed continuous data were presented as mean \pm standard deviation, while categorical data were represented by a number and a percentage. Non-parametric data were presented as a median (interquartile range). All statistical assessments were two-sided and 0.05 was set as the level of statistical significance.

\section{Results}

Thirty-five EN patients included 28 males and 7 females, with ages ranging from 18 and 51 (average age $28.4 \pm 8.4 \mathrm{yrs}$ ). Average TBSA was $67.87 \% \pm 14.87 \%$ and average third-degree burn area was $55.14 \% \pm 14.17 \%$. Twenty-five PN patients included 18 males and 7 females ranging in age between 19 and 61 years (average age $31.5 \pm 10.5$ years). Average TBSA was $68.00 \pm$ $17.84 \%$ and average third-degree burn area was $52.80 \pm 19.53 \%$.

No significant differences in age, gender, TBSA and fullthickness burn area were found between EN and PN groups $(p>0.05)$ (Table 1). Nutrition provision between two groups showed significant differences in total calories, fat, protein, carbohydrates and total liquid volume $(p<0.05)$ (Table 2$)$. Patients in the EN group had higher total calories and carbohydrates and lower fat, protein, total liquid volume than those in the PN group.

The incidence of infection was less in the EN group compared to the PN group $(17.1 \%$ vs $44.0 \% ; p=0.023)$. Additionally, a heightened duration of latency (time to first infection) was observed ( $30.5 \pm 4.7$ days vs $14.4 \pm 2.3$ days; $p<0.001)$, and the infection focus was shortened in 3 cases. Four cases in the EN group were infected by Staphylococcus aureus, while 6 out of 11 cases in the PN group were infected by Enterococcus organisms. A significant difference was observed between the two groups in the duration of antibiotic therapy $(12.5 \pm 3.0$ days in EN group and $19.8 \pm 3.6$ days in the PN group) $(p<0.001)$ (Table 3$)$.

In weekly monitoring of EN and PN groups, the respective mean hemoglobin values $(10.1 \pm 1.3 \mathrm{~g} / \mathrm{L}$ vs $8.3 \pm 1.5 \mathrm{~g} / \mathrm{L} ; p<0.001)$ and respective mean serum albumin values $(44.7 \pm 5.7 \mathrm{~g} / \mathrm{L}$ vs $36.2 \pm 6.9 \mathrm{~g} / \mathrm{L} ; p<0.001)$ for four weeks during the course of treatment revealed that systemic nutritional status and protein synthesis of EN group patients was higher than that of PN group patients; lower hemoglobin and albumin values may also reflect the greater incidence of infection in the PN group. Mortality in the EN group was lower than in the PN group (5.7\% vs $20.0 \%)$, however no significant difference was found $(p=0.117)$ (Table 3$)$.

\section{Discussion}

The results of this study demonstrated that patients with 
Table 1. Patient demographics and basic characteristics

\begin{tabular}{lccc}
\hline Variable & $\begin{array}{c}\text { Enteral nutrition } \\
(n=35)\end{array}$ & $\begin{array}{c}\text { Parenteral nutrition } \\
(n=25)\end{array}$ & $p$ value \\
\hline Age (years) & $28.4 \pm 8.4$ & $31.5 \pm 10.5$ & 0.222 \\
Gender, $n(\%)^{\ddagger}$ & & & $18(72.0)$ \\
$\quad$ Male & $28(80.0)$ & $7(28.0)$ & 0.470 \\
$\quad$ Female & $7(20.0)$ & $68.0 \pm 17.8$ & 0.977 \\
TBSA $(\%)^{\dagger}$ & $67.9 \pm 14.9$ & $52.80 \pm 19.5$ & 0.612 \\
Third degree burn area $(\%)^{\dagger}$ & $55.1 \pm 14.2$ & & \\
\hline
\end{tabular}

Data are displayed as ${ }^{\dagger}$ mean \pm standard deviation and ${ }^{\ddagger}$ number and percentage.

TBSA: total body surface area.

Table 2. Nutrition provision between two groups

\begin{tabular}{|c|c|c|c|}
\hline Variable & $\begin{array}{l}\text { Enteral nutrition } \\
\qquad(n=35)\end{array}$ & $\begin{array}{l}\text { Parenteral nutrition } \\
\qquad(n=25)\end{array}$ & $p$ value \\
\hline Total calories $(\mathrm{kJ})^{\dagger}$ & $16.8 \pm 1.0$ & $14.8 \pm 0.60$ & $<0.001 *$ \\
\hline Fat $(g)^{\dagger}$ & $179.4 \pm 14.4$ & $214.2 \pm 6.4$ & $<0.001 *$ \\
\hline Protein $(g)^{\dagger}$ & $124.7 \pm 4.1$ & $152.3 \pm 6.8$ & $<0.001 *$ \\
\hline Carbohydrates $(\mathrm{g})^{\dagger}$ & $446.3 \pm 12.0$ & $439.2 \pm 8.9$ & $0.015^{*}$ \\
\hline The total liquid volume $(\mathrm{ml})^{\ddagger}$ & $3500(3400,4100)$ & $4500(4000,6000)$ & $<0.001 *$ \\
\hline
\end{tabular}

Data are displayed as ${ }^{\dagger}$ mean \pm standard deviation and ${ }^{\star}$ median and interquartile range.

* Significant difference between the 2 groups, $p<0.05$.

Table 3. Clinical morbidity and mortality between two groups

\begin{tabular}{|c|c|c|c|}
\hline Variable & $\begin{array}{l}\text { Enteral nutrition } \\
\qquad(n=35)\end{array}$ & $\begin{array}{l}\text { Parenteral nutrition } \\
\qquad(n=25)\end{array}$ & $p$ value \\
\hline Mortality, $n(\%)^{\dagger}$ & $2(5.7)$ & $5(20.0)$ & 0.117 \\
\hline Healing time (day) ${ }^{\ddagger}$ & $51.8 \pm 17.9$ & $57.0 \pm 16.2$ & 0.279 \\
\hline Infection, $n(\%)^{\dagger}$ & $6(17.1)$ & $11(44.0)$ & $0.023^{*}$ \\
\hline \multicolumn{4}{|c|}{ Origin of primary infection, $n(\%)^{\dagger}$} \\
\hline Venous intubation & $3(50.0)$ & $1(9.1)$ & \\
\hline Wound & $2(33.3)$ & $2(18.1)$ & 0.053 \\
\hline Unknown & $1(16.7)$ & $8(72.8)$ & \\
\hline \multicolumn{4}{|c|}{ Infective bacteria strain, $n(\%)^{\dagger}$} \\
\hline Staphylococcus aureus & $4(66.7)$ & $1(9.1)$ & \\
\hline Enterococcus & $0(0.0)$ & $6(54.6)$ & \\
\hline Bacteroides & $0(0.0)$ & $2(18.1)$ & \\
\hline Pseudomonas & $2(33.3)$ & $1(9.1)$ & \\
\hline E. coli & $0(0.0)$ & $1(9.1)$ & \\
\hline Latency (day) $)^{\ddagger}$ & $30.5 \pm 4.7$ & $14.4 \pm 2.3$ & $<0.001 *$ \\
\hline Usage of antibiotics (day) ${ }^{\ddagger}$ & $12.5 \pm 3.0$ & $19.8 \pm 3.6$ & $<0.001 *$ \\
\hline Hemoglobin $(\mathrm{g} / \mathrm{L})^{\ddagger}$ & $10.06 \pm 1.34$ & $8.29 \pm 1.45$ & $<0.001 *$ \\
\hline Albumin $(g / L)^{\ddagger}$ & $44.74 \pm 5.73$ & $36.20 \pm 6.88$ & $<0.001 *$ \\
\hline
\end{tabular}

Data are displayed as ${ }^{\dagger}$ number (percentage) and ${ }^{*}$ mean \pm standard deviation.

* Significant difference between the two groups, $p<0.05$.

extensive burns who received enteral nutrition in the early postburn period experienced a lower incidence of systemic infection than subjects receiving parenteral nutrition only. In the EN group, the latency period between burn injury and first infection was longer and a clear infection focus was usually found, allowing timely, effective treatment. In contrast, PN patients had a higher early post-burn infection rate and a shorter latency period.

Extensive burns result in edema of the GI tract mucosa, intestinal dysfunction, increased gut permeability and energy deficiency in the intestinal mucosa. This leads to mucosal atrophy and disturbs patients' nutrition, metabolism, immunity and ability to heal. $^{(2-5)}$ In addition, usage of broad-spectrum antibiotics and prolonged fasting alters intestinal microecology and colonization of pathogens. Translocation of intestinal bacteria to the GI tract and breakdown of the intestinal barrier elicit enterogenic infec- tion. (2) The hypermetabolic state induced by burns increases patients' risk for malnutrition, resulting in weight loss, poor wound healing, muscle wasting and immunosuppression that can last long after the burn, making nutritional support vital to reduce post-burn infection, recovery time and long-term sequelae. ${ }^{(6)}$

Protection of the GI tract and its function reduces hypermetabolism, systemic infection, and septicemia in patients with extensive burns; ${ }^{(3,6,12)}$ compatible with these findings, we found that early post-burn EN helps prevent injury to the GI barrier, improves blood flow, alleviates ischemic and hypoxic mechanical damage and restores microecological balance within the GI tract. The lower infection rate and reduced usage of antibiotics in the EN group strongly suggest that post-burn EN facilitates suppression of post-burn infection. Consistent with our findings, Hart et al. ${ }^{(13)}$ found that aggressive feeding attenuated muscle catabolism and 
improved infection outcomes after burn injuries in children, especially when combined with early surgical excision; also early, aggressive treatment did not decrease energy expenditure but did support protein catabolism compared to delayed enteral feeding. A study focusing on effects of early versus delayed EN on clinical, nutritional and endocrine outcomes after severe burns found that initiating EN within hours after burn injury reduces caloric deficits, stimulates insulin secretion and protein retention in the early treatment phase, although it was not associated with measureable improvement in endocrine status or reduced hypermetabolism, morbidity or mortality. ${ }^{(14)}$

In burned patients receiving parenteral nutrition only, intestinal mucosa atrophy resulting from lack of food stimulus may aggravate damage to intestinal barrier function, increasing intestinal bacteria translocation, systemic inflammatory response syndrome, and organ dysfunction. ${ }^{(15)}$ These risks reinforce the idea that burn patients should be evaluated for early EN on admission to the burn unit. ${ }^{(7,14)}$ Those with unstable factors such as a systemic disorder should not receive nutritional support until stabilized. Also, if any gastrointestinal reaction develops signaling intestinal intolerance, EN should be suspended for a period of time before restarting. ${ }^{(4)}$

Regarding energy requirements, Chan, $M$ and Chan, $G^{(6)}$ indicated that metabolic rates in burn patients are often double normal rates and that demand for more than 5,000 calories daily is not uncommon. However, they caution that administration of excess calories is associated with increased metabolic rate, hyperglycemia, liver dysfunction and increased $\mathrm{CO}_{2}$ production. ${ }^{(6)}$ Consequently, we met individual calorie needs using an amount slightly lower than patients' calculated value, aiming to achieve better glucose control and prevent infection-related complications. Our strategy is to gradually improve quality and quantity of food intake according to each patient's recovery rate, GI tract capacity and recovery of peristalsis. We also strive to increase GI tract tolerance by first introducing warm liquids (e.g., nutrient-rich liquid reserved from cooking rice or millet in water), which helps maintain GI tract tension, increase blood flow and reduce injury, thereby accelerating functional recovery. In our experience, nutritional content should be retained in the GI tract by feeding as early as possible post-burn to prevent atrophy of disuse and accelerate recovery of GI function. Under normal conditions, the intestinal mucosa functions as a local defense barrier that prevents bacteria from spreading to extraluminal tissues and organs. ${ }^{(2)}$ This translocation mechanism becomes overwhelmed in the immunosuppressed post-burn patient, further complicated by administration of antibiotics. Enteral feeding prevents exaggerated trans-

\section{References}

1 Mass Casualties. Burns. Centers for Disease Control and Prevention. Atlanta, Georgia USA. 2006; http://emergency.cdc.gov/masscasualties/burns.asp. Accessed 15 Dec 2009.

2 Magnotti LJ, Deitch EA. Burns, bacterial translocation, gut barrier function, and failure. J Burn Care Rehabil 2005; 26: 383-391.

3 Shi ZH. The gastrointestinal dysfunction and multiple organ dysfunction syndrome after severe burns. Med Clin Res 2004; 21 : 510-514.

4 Guo L, Shi ZH, Zhang HB, and et al. The influence of burn and endotoxin/ LPS on gastrointestinal motility in guinea pigs. Chin J Burns 2005; 21: 350352.

5 Peng X, Tao LH, You ZY, Wang P, Wang SL. The relationship between intestinal damage and enterogenous hypermetabolism after severe burn. Acta Academiae Medicinae Militaris Tertiae 2003; 25: 1745-1747.

6 Chan MM, Chan GM. Nutritional therapy for burns in children and adults. Nutrition 2008; 25: 261-269.

7 Wasiak J, Cleland H, Jeffery R. Early versus late enteral nutritional support in adults with burn injury: a systematic review. J Hum Nutr Diet 2007; 20: 75-83.

8 Peng X, You ZY, Wang P, Wang SL. Impact of different nutritional support routes on enterogenic hypermetabolism after burn. Parenteral \& Enteral location by limiting mucosal injury; this maintains mucosal mass and supports intestinal structural integrity, stimulating epithelial cell proliferation and blood flow to the gut, and promoting production of beneficial enzymes and various endogenous trophic agents. ${ }^{(2)}$ Recovery of peristalsis also depends on functional integrity of the intestinal mucosa. Intestinal mucosal cells have considerable volume, a high updating speed and express a large energy demand. The support of EN allows intestinal mucosal cells to supply the body with energy and raw materials to repair damage, and simultaneously provides substantial energy directly to intestinal cells themselves. Hence, early feeding after burn injury is important in maintaining the intestinal barrier and rehabilitating intestinal damage post-burn..$^{(5,8)}$

Our study is limited by lack of a specific mechanism by which to examine details of post-burn enteral nutrition and its effects on intestinal metabolism in order to validate our clinical findings. Although we used weekly monitoring of hemoglobin and albumin values to provide an overview of systemic nutrition and protein synthesis during treatment, the study lacked definitive markers of nutritional status. Nevertheless, increases in hemoglobin values did reflect improved blood oxygen carrying capacity, which helped to alleviate ischemia and hypoxia and increase the antiinfective ability of tissues. Also, hemoglobin and albumin values were markedly lower in the PN group, most likely reflecting the higher incidence of infection in this group. Future study is needed to corroborate our results, focusing on post-burn mechanical destruction of the intestinal mucosa and alterations of the intestinal microecology that might be prevented or managed by employing early GI nutrition. We plan to conduct a simple randomized controlled trial (RCT) on early enteral feeding that would eliminate risk of biases and provide a more definitive evaluation of the benefits of early enteral feeding.

Enteral nutrition in the early post-burn period can decrease patients' infection rate and suppress systemic infection after extensive burns. Further study is needed to help establish more definitive guidelines for clinical practice.

\section{Abbreviations}

$\begin{array}{ll}\text { EN } & \text { enteral nutrition } \\ \text { GI } & \text { gastrointestinal } \\ \text { PN } & \text { parenteral nutrition } \\ \text { RCT } & \text { randomized controlled trial } \\ \text { TBSA } & \text { total body surface area }\end{array}$

Nutrition 2005; 12: 159-161.

9 Peng YZ, Yuan ZQ, Xiao GX. Effects of early enteral feeding on the prevention of enterogenic infection in severely burned patients. Burns 2001; 27: 145-149.

10 Raff T, Hartmann B, Germann G. Early intragastric feeding of seriously burned and long-term ventilated patients: a review of 55 patients. Burns 1997; 23: $19-25$.

11 Lee JO, Benjamin D, Herndon DN. Nutrition support strategies for severely burned patients. Nutr Clin Pract 2005; 20: 325-330.

12 Wang SL, Wang SL, Li A. Stress and enteral nutritional support after burn/ injury. Chinese J Plastic Surg Burns 1998; 14: 225-227.

13 Hart DW, Wolf SE, Chinkes DL, and et al. Effects of early excision and aggressive enteral feeding on hypermetabolism, catabolism, and sepsis after severe burn. J Trauma 2003; 54: 755-761.

14 Gottschlich MM, Jenkins ME, Mayes T, Khoury J, Kagan RJ, Warden GD. The 2002 Clinical Research Award. An evaluation of the safety of early vs delayed enteral support and effects on clinical, nutritional, and endocrine outcomes after severe burns. J Burn Care Rehabil 2002; 23: 401-415.

15 Wang ZG. Study of attention to systemic inflammatory response syndrome. Chinese J Int Med 1999; 38: 151. 